\title{
Flexible quantum tokens in spacetime
}

\author{
Adrian Kent ${ }^{1,0}{ }^{1, *}$ and Damián Pitalúa-García ${ }^{1, \dagger}$ \\ ${ }^{1}$ Centre for Quantum Information and Foundations, DAMTP, Centre for Mathematical Sciences, University of Cambridge, \\ Cambridge CB3 OWA, United Kingdom \\ ${ }^{2}$ Perimeter Institute for Theoretical Physics, Waterloo, Ontario, Canada N2L $2 Y 5$
}

(Received 21 August 2019; revised manuscript received 19 November 2019; accepted 5 December 2019; published 10 February 2020)

\begin{abstract}
S-money [A. Kent, Proc. R. Soc. A 475, 20190170 (2019)] schemes define virtual tokens designed for networks with relativistic or other trusted signaling constraints. The tokens allow near-instant verification and guarantee unforgeability without requiring quantum state storage. We present refined two stage S-money schemes. The first stage, which may involve quantum information exchange, generates private user token data. In the second stage, which need only involve classical communications, users determine the valid presentation point, without revealing it to the issuer. This refinement allows the user to determine the presentation point anywhere in the causal past of all valid presentation points. It also allows flexible transfer of tokens among users without compromising user privacy.
\end{abstract}

DOI: 10.1103/PhysRevA.101.022309

\section{INTRODUCTION}

Money and other type of tokens allow a user to access a resource, while guaranteeing to the issuer that the tokens cannot be forged. Quantum money and quantum token schemes [1-4] can theoretically guarantee unforgeability with unconditional security. However, standard quantum money and quantum token schemes are impractical with current technology because they require long-term quantum state storage. Quantum memories cannot currently store quantum states reliably for more than a fraction of a second. Although longer term quantum memories will presumably be developed in future, it is unclear whether (or when) quantum memory technology will ever be competitive in price and practicality with classical alternatives.

S-money [5] schemes [6-9] offer alternatives to quantum money that do not require quantum memory and can respond more flexibly to generalized summoning tasks [6]. However, many other theoretical and practical questions about the relative advantages, implementability and resource costs of S-money and quantum money token schemes remain to be addressed. In our view, both technologies are potentially promising and merit further refinement and development.

In this paper we describe refinements of S-money schemes that improve their flexibility and transferability. We focus on a single round implementation of a simple quantum S-money token scheme in order to illustrate the essential ideas; it is straightforward to extend these to multiple rounds, to other types of S-money token, to schemes involving subtokens, and to more complex scenarios. We also simplify the discussion by focusing on an S-money scheme that guarantees unforgeability and future privacy for the user, without the extra cryptographic layer required to ensure past privacy; again, the extension is straightforward.

\footnotetext{
*apak@damtp.cam.ac.uk

†D.Pitalua-Garcia@damtp.cam.ac.uk
}

\section{FLEXIBLE S-MONEY}

We follow the standard scenario for S-money [6]. The issuer (Bob the bank) consists of a network of local agents based at secure sites, with authenticated and secure communication links, working in collaboration and with mutual trust. Similarly, the user (Alice the acquirer) consists of a network of local agents based at secure sites, with authenticated and secure communication links, working in collaboration and with mutual trust. The user and issuer agree to implement the scheme but do not trust one another to behave honestly. The user's secure sites do not overlap with the issuer's. The user and issuer agree on a reference frame, where spacetime coordinates are defined. The user and issuer agree in advance on a set of presentation points $Q_{i}$, where the user can present an S-money token to the issuer in order to access a resource. The scheme begins at some start point $P$ with $P \prec Q_{i}$ (that is, $P$ is in the causal past of $Q_{i}$ ) for all $i$. To be precise, both $P$ and the $Q_{i}$ actually represent localized spacetime regions containing separate secure sites for the user and issuer, with a time window allowing prescribed communications between these sites. Because the spatial and/or temporal separations between these regions are large (in the relevant rest frame) compared to those of the regions, we can think of them as effectively points on a spacetime network. The token scheme is initiated by classical and/or quantum information communications between user and issuer agents at $P$. To consider transferability of tokens we refer to two or more users (Alice ${ }_{1}$, Alice $\left._{2}, \ldots\right)$. In this case each user has his or her own separate secure site at each relevant network point.

\section{A. Bit-string coordination and S-money schemes}

These schemes are based on bit-string coordination [6] protocols between the user and issuer. Bit-string coordination is an intrinsically relativistic task in which the user inputs, or commits, a string $b$ at some point in spacetime and later unveils by giving classical data to the issuer to show that her input 
was $b$. A secure implementation of bit-string coordination satisfies two security properties: binding and hiding. We say a protocol is binding if it guarantees to the issuer that if the user successfully proves having input $b$, then she cannot also prove (either at the same spacetime point or elsewhere) having input a different string $b^{\prime}$. We say a protocol is hiding if it guarantees to the user that the issuer cannot know $b$ until it is unveiled by the user. Bit-string coordination is similar to but weaker than bit-string commitment, which also needs to guarantee to the issuer that the user committed to her input at some specified start point. In this paper we apply the language of bit-string commitment to bit-string coordination, as specified above.

In the S-money schemes [6,7] we consider here, the user defines her bit string by choices of measurements on quantum states sent by the issuer. The schemes and our refinements are secure regardless of the user's technological limitations. However, to simplify the discussion, we will assume the user is technologically limited and has no quantum state storage, which effectively forces her to carry out these measurements on receipt. Given this limitation, the bit-string coordination protocol effectively becomes a bit-string commitment protocol, with the commitment being made when the states are measured. Specifically, the commitment phase for each bit in the string is that of the BB84 bit commitment protocol of Ref. [10], whose security was analyzed in Refs. [10-13]. This protocol was implemented experimentally [13] with a modification involving precommitment to a random string in order to allow practical implementation without requiring long distance quantum communication. This technique was used also used in practical protocols for spacetime-constrained oblivious transfer $[14,15]$. We use variations on this idea here.

\section{Example of a bit coordination scheme}

We present a simple bit coordination scheme given in Ref. [7], which is based on the bit commitment protocol of Ref. [10], to illustrate why bit-string coordination and bitstring commitment are different tasks.

Let $\{|0\rangle,|1\rangle,|+\rangle,|-\rangle\}$ be the set of Bennett-Brassard 1984 (BB84) states, where $| \pm\rangle=\frac{1}{\sqrt{2}}(|0\rangle \pm|1\rangle)$ and where the states $|0\rangle$ and $|1\rangle$ are orthonormal. We define the following qubit states:

$$
\left|\phi_{0}^{0}\right\rangle=|0\rangle, \quad\left|\phi_{1}^{0}\right\rangle=|1\rangle, \quad\left|\phi_{0}^{1}\right\rangle=|+\rangle, \quad\left|\phi_{1}^{1}\right\rangle=|-\rangle .
$$

Let $n \in \mathbb{N}$ be a security parameter. For strings $\mathbf{r}, \mathbf{s} \in\{0,1\}^{n}$, let their respective bit entries be denoted by $r_{l}, s_{l} \in\{0,1\}$, for $l \in[n]$, where we define $[n]=\{1,2, \ldots, n\}$. We define the state

$$
\left|\Psi_{\mathbf{r}, \mathbf{s}}\right\rangle=\bigotimes_{l \in[n]}\left|\phi_{r_{l}}^{s_{l}}\right\rangle,
$$

for $\mathbf{r}, \mathbf{s} \in\{0,1\}^{n}$.

The issuer generates a state $|\Psi\rangle=\left|\Psi_{\mathbf{r}, \mathbf{s}}\right\rangle$ of $n$ qubits, with $\mathbf{r}$ and $\mathbf{s}$ chosen randomly from $\{0,1\}^{n}$, and sends $|\Psi\rangle$ to the user at a spacetime point $P$. The issuer sends the labels $l$ of the transmitted qubits, for $l \in[n]$. The user commits to the bit $b=0$ by measuring all received qubits in the computational basis $(\{|0\rangle,|1\rangle\})$ or to the bit $b=1$ by measuring all received qubits in the Hadamard basis $(\{|+\rangle,|-\rangle\})$. Let $y_{l}$ be the bit measurement outcome corresponding to the qubit labeled by $l$, for $l \in[n]$, and let $\mathbf{y}=\left(y_{1}, \ldots, y_{n}\right)$. The user unveils at some point $Q \succ P$ by giving the bit $b$ and the string of outcomes $\mathbf{y}$ to the issuer. In the ideal case that errors are not tolerated, the issuer accepts $b$ as valid if $y_{l}=r_{l}$ for all $l \in[n]$ satisfying $s_{l}=b$. This means, for example, that if the user unveils $b=0$, and he is being honest, he measured all qubits in the computational basis; hence, the user obtains the correct outcomes for all qubits prepared by the issuer in the computational basis. In a realistic case, the issuer accepts $b$ as valid if it holds that $y_{l} \neq r_{l}$ for no more than $\gamma\left|\Omega_{b}\right|$ qubits with labels $l \in \Omega_{b}$, where $\Omega_{b}=\left\{l \in[n] \mid s_{l}=b\right\}$, for a predetermined small-enough $\gamma \in\left(0, \frac{1}{2}\right)$.

This bit coordination protocol is hiding because the user does not give any information to the issuer about her chosen bit $b$ before she unveils. It is binding because the user cannot, at any two points $Q, Q^{\prime}$, give the issuer two $n$-bit strings $\mathbf{y}=$ $\left(y_{1}, \ldots, y_{n}\right)$ and $\mathbf{z}=\left(z_{1}, \ldots, z_{n}\right)$, corresponding, respectively, to unveiling $b=0$ and $b=1$, that satisfy both conditions (1) $y_{l} \neq r_{l}$ for no more than $\gamma\left|\Omega_{0}\right|$ qubits with labels $l \in \Omega_{0}$ and (2) $z_{l} \neq r_{l}$ for no more than $\gamma\left|\Omega_{1}\right|$ qubits with labels $l \in \Omega_{1}$ for a predetermined small-enough $\gamma \in\left(0, \frac{1}{2}\right)$. This property follows from the security analyses given in Refs. [10-13,16].

However, this does not define an unconditionally secure bit commitment protocol, since the issuer receives no useful guarantee on the spacetime region where the user chose $b$. For example, a technologically unlimited user could store all received states in a perfect quantum memory, send them to $Q$, and measure them there in the relevant basis just before unveiling $b$. The issuer is guaranteed that the bit was chosen at some point $Q^{\prime} \preceq Q$ but not that it was chosen at $P$ or any other specified point $Q^{\prime} \prec Q$.

\section{B. Limitations of previous S-money schemes \\ 1. Initiation time}

The bit coordination protocol just discussed can be repeated $N$ times, giving an $N$-bit-string coordination protocol [6]. This defines a simple S-money token protocol [6,7], in which the bit string labels the point $Q_{i}$ (for $i \in\{0,1\}^{N}$ ) at which the token may validly be presented. The S-money scheme is initiated by the issuer sending a string of quantum states to the user at the start point. In the ideal version, these states are pure qubits drawn randomly from the four BB84 states, sent along a lossless channel, and the user carries out perfect BB84 basis measurements on each state. In practical embodiments with photonic systems, this quantum communication step involves generating a sequence of weak photon pulses, with some errors in the polarizations chosen, some losses, and some measurement errors. Depending on the experimental parameters, a reasonable level of security may require the transmission and measurement of a large number of photon pulses. With current technology, this could take at least a few seconds. In an S-money scheme, this would require the user to choose her presentation point $Q_{b}$ at least a few seconds in the past of $Q_{b}$.

This is a significant and undesirable limitation, since Smoney schemes are intended for application in relativistic scenarios where time is critical and where decisions about when and where to present the S-money are ideally made as 
flexibly as possible (and in particular as late as possible) on the basis of incoming information received at various spacetime points.

Quantum technology is developing rapidly, and it may be possible to generate, send, and measure enough quantum states to initiate secure tokens within very short time intervals in the future. Nonetheless, we think it likely that the alternatives we discuss below, which require only classical communications at the critical decision point(s), will continue to be faster and cheaper. Our discussion and illustrations rely only on this assumption, rather than on specific quantitative estimates.

\section{Transferability and transfer time}

A related limitation of the S-money schemes [6,7], as presented, is that the process of transferring tokens either lacks flexibility or requires a delay to initiate a new token.

Suppose that $B$ issues a token to $A_{1}$, by sending a string of quantum states within a (suitably extended) spacetime region $P$. We assume that $A_{1}$ has no long-term quantum memory, since this is the underlying motivation for the schemes. Generating a token thus requires $A_{1}$ to measure the BB84 states sent by $B$ as soon as she receives them, in bases that label her chosen presentation point $Q_{b}$.

Suppose now that $A_{1}$ later wishes to transfer the token to $A_{2}$ at some point $Q$, where $P \prec Q \prec Q_{b}$. Two options are considered in Ref. [6] as follows:

(1) $A_{1}$ may give $A_{2}$ the rights to a token which is valid only at her already-determined presentation point, $Q_{b}$. She can do this by giving $A_{2}$ at $Q$ her measurement outcomes and the number $b$, along with a digitally signed classical message stating that $A_{1}$ relinquishes her token rights and $A_{2}$ is now the owner. $A_{2}$ can send this signed message to a local agent of $B$ at or near $Q$, who informs all agents of $B$ in his causal future. These agents will then no longer accept the token from any agent of $A_{1}$ but will accept it from $A_{2}$.

(2) $A_{1}$ may give $A_{2}$ the rights to the token at $Q$ in a way that allows $A_{2}$ to choose a new valid presentation point, $Q_{b^{\prime}} \succ Q$. She can do this by giving $A_{2}$ a digitally signed message stating that she relinquishes her token rights and that the token is no longer valid. $A_{2}$ sends this to a local agent of $B$, as above. In this case, the local agents of $B$ and $A_{2}$ need to initiate a replacement token. This requires $B$ 's local agent to send a string of quantum states to $A_{2}$ 's local agent, who measures them, following the protocol above.

The first option has the advantage that transfers require only classical communications and so can be completed relatively quickly. However, it is inflexible; $A_{2}$ is restricted to presenting the token at the point $Q_{b}$ previously chosen by $A_{1}$.

The second has the advantage of flexibility; $A_{2}$ may choose a new presentation point, which may be any valid presentation point within the causal future of the transfer point $Q$. However, it requires a new round of quantum communications, with the associated delay.

\section{Flexible S-money schemes}

In this paper, we propose an implementation of S-money that has none of the above disadvantages. The scenario we envisage requires that users may realize they may potentially want to acquire and use S-money well in advance of actually doing so. This seems generally realistic. For example, traders who plan high-value high-speed trades on the global financial network expect to be registered, authenticated, and licensed, to set up infrastructure, and so on, before they begin trading: They do not expect to be able to show up on the network unannounced and uncredentialled and instantly trade.

We thus propose that such users go through information exchanges with the bank, in which the bank sends strings of quantum states which the user measures, in some starting region $S$, well before they might acquire or use $S$-money. These exchanges define bit-string coordination protocols which, if the user is unable to store quantum states, effectively commit the user to long random bit strings $x$. To simplify the discussion, we will assume the users have this technological limitation. However, this is not required for the security of the schemes, which (like the original schemes discussed above) require only secure bit-string coordination, not bit-string commitment. The bank's data received from each user's protocol are shared with all the bank's local agents but kept private from other users.

To acquire an S-money token, a user $A$ then needs only to communicate classically with the bank at some later point $P_{A}$. They first agree a set of valid presentation points $\left\{Q_{i}: i \in\right.$ $S$ \}. Here $S$ satisfies $2^{M-1}<|S| \leqslant 2^{M}$, each $Q_{i} \succeq P_{A}$, and they agree a standard convention for the labeling of the $Q_{i}$ by bit strings $i$. They also agree which previously unused length $M$ segment $x$ of the user's committed bit string will be associated with this token, by labeling the relevant bits. (The user keeps the value of $x$ secret here.) They may also at this point agree the price to be paid (e.g., in some standard currency) for the S-money token and perhaps process the payment. The bank's local agent sends all the information received to the bank's agents at each $Q_{i}$.

The user may then later decide where the token will be valid and commit to this decision by choosing $b \in S$ at any network point $P_{D}$ such that $P_{A} \preceq P_{D} \preceq Q_{i}$ for all $i \in S$. The user's local agent at $P_{D}$ commits this to the bank's local agent by sending him the string $m=x \oplus b$, and the bank's local agent sends this to the bank's agents at each $Q_{i}$, where " $\oplus$ " denotes sum modulo two.

Finally, at $Q_{b}$, the user's local agent unveils the commitment to $x$ by sending her commitment data to the bank's local agent. The bank's local agent verifies the commitment and that $m \oplus x=b$; if these tests are passed, then the S-money token is validated at $Q_{b}$, and the user is given whatever resources were agreed there.

\section{Example}

In the token scheme presented in Ref. [7] the issuer transmits a quantum state $|\Psi\rangle$ to the user, chosen from a predetermined set. At reception of $|\Psi\rangle$, the user chooses the number $b$ labeling the presentation point $Q_{b}$ by applying on $|\Psi\rangle$ a quantum measurement $M_{b}$ that belongs to a predetermined set, obtaining a classical measurement outcome y [17]. The user presents the token at $Q_{b}$ by presenting the classical measurement outcome $\mathbf{y}$ at $Q_{b}$. The issuer validates the token at $Q_{b}$ if he verifies that the data $\mathbf{y}$ received from the user at $Q_{b}$ corresponds to a valid measurement outcome of the quantum measurement $M_{b}$ applied on $|\Psi\rangle$. 
The following example to implement the previous token scheme is given in Ref. [7]. Let the presentation points $Q_{i}$ be labeled by strings $i=\left(i_{1}, \ldots, i_{M}\right) \in\{0,1\}^{M}$ for an integer $M$ predetermined by the user and issuer. Let $b=\left(b_{1}, \ldots, b_{M}\right) \in$ $\{0,1\}^{M}$ be the string that labels the user's chosen presentation point $Q_{b}$. The user and issuer engage in $M$ parallel protocols of the bit coordination scheme presented in Sec. II A 1 . The $k$ th bit coordination protocol defines the bit entry $b_{k}$ of the user's string $b$, for $k \in[M]$. Thus, more precisely, the issuer generates a state $|\Psi\rangle=\left|\Psi_{\mathbf{r}, \mathbf{s}}\right\rangle$ of $n M$ qubits given by

$$
\left|\Psi_{\mathbf{r}, \mathbf{s}}\right\rangle=\bigotimes_{(k, l) \in[M] \times[n]}\left|\phi_{r_{l}^{k}}^{s_{l}^{k}}\right\rangle,
$$

where $\mathbf{r}$ and $\mathbf{s}$ are chosen randomly from $\{0,1\}^{M n}$ by the issuer and where $r_{l}^{k}$ and $s_{l}^{k}$ denote the respective bit entries with labels $(k, l)$ of the strings $\mathbf{r}$ and $\mathbf{s}$, for $k \in[M]$ and $l \in[n]$. The issuer sends to the user the state $|\Psi\rangle$ and the labels $(k, l)$ of the transmitted qubits for $k \in[M]$ and $l \in[n]$. The user's measurement $M_{b}$ consists in measuring the received qubit with label $(k, l)$ in the computational basis $(\{|0\rangle,|1\rangle\})$ if $b_{k}=0$ or in the Hadamard basis $(\{|+\rangle,|-\rangle\})$ if $b_{k}=1$, respectively, for $l \in[n]$ and for $k \in[M]$.

In our flexible S-money scheme, at reception of the token from the issuer, the user applies the quantum measurement $M_{x}$ on the quantum state $|\Psi\rangle$ received from the issuer, where $x$ is a random $M$-bit string chosen by the user. The user obtains a classical measurement outcome $\mathbf{y}$. Then the user chooses the $M$-bit string $b$ labeling her presentation point $Q_{b}$ and sends $m=x \oplus b$ to the issuer at a point in the causal future of the point where she completed the measurement $M_{x}$ and within the intersection of the causal pasts of all the presentation points. The user gives the token $\mathbf{y}$ to the issuer at $Q_{b}$. The issuer validates the token if he verifies that $\mathbf{y}$ is a statistically plausible measurement outcome of the quantum measurement $M_{m \oplus b}$ applied to $|\Psi\rangle$. This is illustrated in Fig. 1.

\section{Security of flexible S-money schemes}

\section{Unforgeability}

A refined S-money scheme based on a bit-string coordination protocol that is binding satisfies the property of unforgeability, according to which the user cannot have two or more tokens validated at two or more presentation points, as we discuss. In a cheating strategy by the user trying to present tokens at two different presentation points $Q_{i_{1}}$ and $Q_{i_{2}}$, the user must be able to present unveiling data that correspond to a valid commitment to a number $x_{1}$, satisfying $m \oplus x_{1}=i_{1}$, and also to a number $x_{2}$, satisfying $m \oplus x_{2}=i_{2}$. Since $i_{1} \neq i_{2}$, we have that $x_{1} \neq x_{2}$. However, if the bit-string coordination protocol is binding, then, by definition, if the user presents unveiling data that corresponds to a valid commitment to a number $x_{1}$, then the user cannot also present unveiling data that correspond to a valid commitment to a number $x_{2} \neq x_{1}$. Thus, the property of unforgeability follows.

\section{Future privacy for the user}

A refined S-money scheme based on a bit-string coordination protocol that is hiding and where $x$ is chosen randomly and securely by the user satisfies the property of future privacy

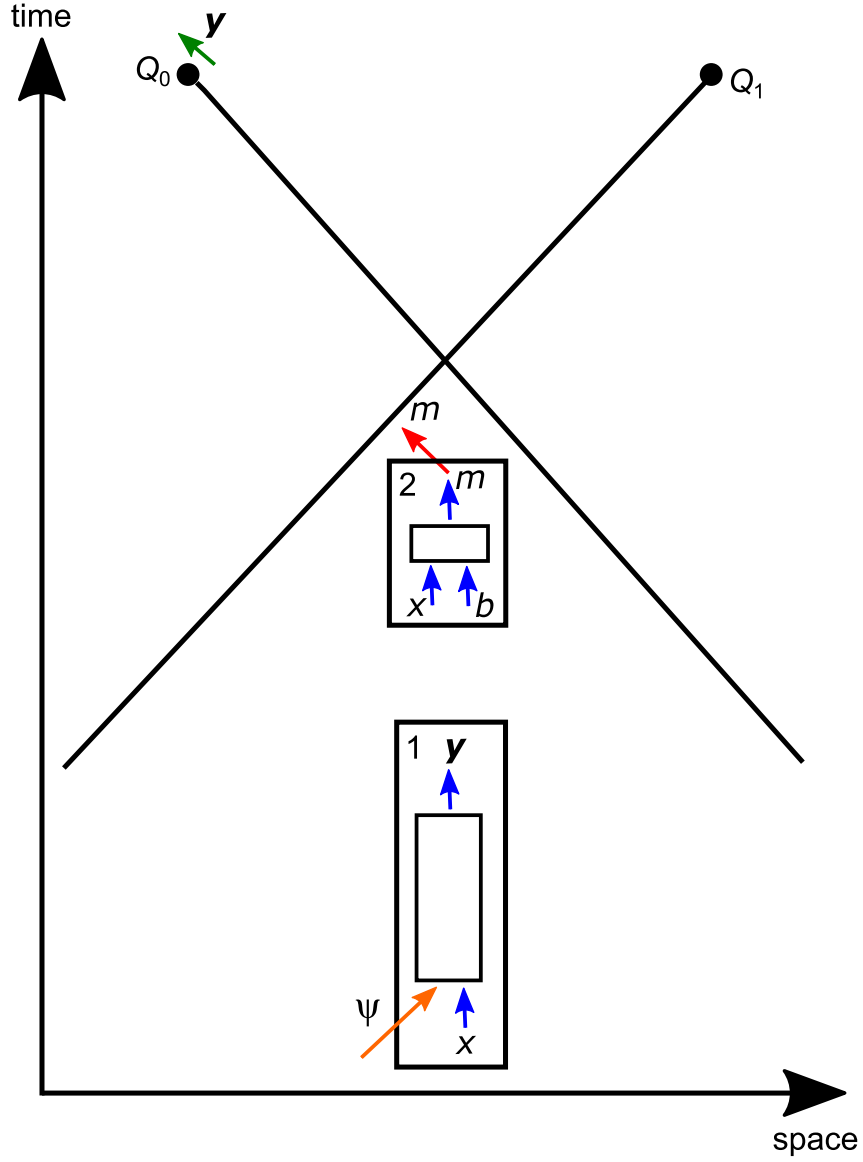

FIG. 1. Our flexible S-money scheme described in the main text is illustrated in $1+1$-dimensional Minkowski spacetime for the case of two presentation points $Q_{0}$ and $Q_{1}$ (black dots). We use units in which the speed of light is unity. The black lines denote light rays. Bob's transmission of the quantum state $|\Psi\rangle$ to Alice is illustrated with the large orange diagonal arrow. The measurement on $|\Psi\rangle$ by Alice is represented by the rectangle labeled " 1. " The generation of the classical message $m=x \oplus b$ by Alice is represented by the square labeled "2." Alice's transmission of $m$ to Bob is illustrated with the red diagonal arrow. The transmission of the token consists in Alice giving her measurement outcome $\mathbf{y}$ to Bob at $Q_{b}$ (green diagonal arrow). The case $b=0$ is illustrated. The box labeled "1" illustrates Alice's generation of the random string $x$ and the application of the measurement $M_{x}$ on the received quantum state $|\Psi\rangle$, which outputs a classical measurement outcome y. The box labeled " 2 " illustrates Alice's computation of the message $m=x \oplus b$, where $b$ labels her chosen presentation point $Q_{b}$. Alice's inputs, $x$ and $b$, and outputs, $m$ and $\mathbf{y}$, are represented by the blue vertical arrows.

for the user, according to which the issuer cannot obtain any information about the presentation point $Q_{b}$ chosen by the user before the token is presented by the user, as we discuss. In a cheating strategy by the issuer trying to obtain some information about the number $b$ labeling the presentation point $Q_{b}$ chosen by the user, the issuer may try to learn some information about the number $x$ to which the user commits at the reception of the token in the bit-string coordination protocol and use this obtained information together with the message $m=x \oplus b$ received from the user to obtain some information about $b$. However, if we assume that the bit-string 
coordination protocol is hiding, then, by definition, the issuer cannot know the value of $x$ before it is unveiled by the user. Therefore, assuming that $x$ is chosen randomly and securely by the user, the issuer cannot obtain any information about $b$ from the message $m=x \oplus b$ before $x$ is unveiled by the user. Thus, future privacy for the user follows.

\section{TRANSFERRING S-MONEY TOKENS}

Our refinement allows S-money tokens to be traded and transferred between users quite simply and flexibly. Suppose the first user $A_{1}$ has acquired a token at point $P$, associated with her committed bit string $x_{1}$, and now wishes to transfer it to a second user $A_{2}$ at point $P_{T}$, where $P \preceq P_{T} \preceq Q_{i}$ for all $i$. In the simplest version, $A_{1}$ 's local agent at $P_{T}$ simply gives $A_{2}$ 's local agent all the data defining the labels of the presentation points in $S$ and an authenticated digitally signed message transferring her rights in the token. $A_{2}$ verifies this message and registers the transfer with the bank's local agent, agreeing to associate to the token a labeled segment $x_{2}$ of $A_{2}$ 's precommitted random string. The local agents of $A_{1}$ and $A_{2}$ may also at this point agree the price to be paid (e.g., in some standard currency) for the S-money token transfer and perhaps process the payment. The bank's local agent notifies all agents at points $Q_{i}$ of the transfer and of the labeled string segment.

Now $A_{2}$ may decide the valid presentation point $Q_{b}$ at any point $P_{D}$ such that $P_{T} \preceq P_{D} \preceq Q_{i}$ for all $i$ and commit to this by sending the string $m_{2}=x_{2} \oplus b$ to Bob. She may present the token at $Q_{b}$ by sending all the commitment data for $x_{2}$ (which comes from her own commitments) to Bob's local agent there. Bob's local agent validates both commitments and accepts the token if the commitments are validly unveiled and $m_{2} \oplus x_{2}=b$.

Alternatively, instead of deciding a presentation point, $A_{2}$ may transfer the token to $A_{3}$ at some transfer point $P_{T^{\prime}} \succeq P_{T}$ by sending all the relevant data and her own authenticated digitally signed transfer message, and so on.

Each user $A_{i}$ is guaranteed future privacy regarding their token presentation point, since when they commit to the valid presentation point $Q_{b}$ at some point $P_{D}$ they send a string $m_{i}=$ $x_{i} \oplus b$, where $x_{i}$ is a committed private random string.

The key advantage is summarized schematically in Fig. 2.

\section{DELAYING THE CHOICE OF PRESENTATION POINT}

This technique extends to allow the user to make a sequence of decisions and commitments at separate points in spacetime that collectively decide the presentation point, while still guaranteeing to the issuer that there can be no more than one valid presentation point.

Let $P_{D_{i}}$ for $i=1, \ldots, N$ be a set of decision points, with at least one of them, $P_{D_{1}}$, in the causal past of all valid presentation points. Let $\left\{Q_{i}: i \in S\right\}$ be the set of presentation points. The user may decide and commit at $P_{D_{1}}$ to restrict the valid presentation point to lie in a subset $\left\{Q_{i}: i \in S_{1}\right\}$, sending the issuer a description of the subset $S_{1}$ committed using data from their precommitted random string $x$. Let $P_{D_{2}}$ lie in the causal past of all $Q_{i}$ with $i \in S_{1}$. The user may decide and commit at $P_{D_{2}}$ to restrict the valid presentation point to lie in a subset $\left\{Q_{i}: i \in S_{2}\right\}$, sending the issuer a

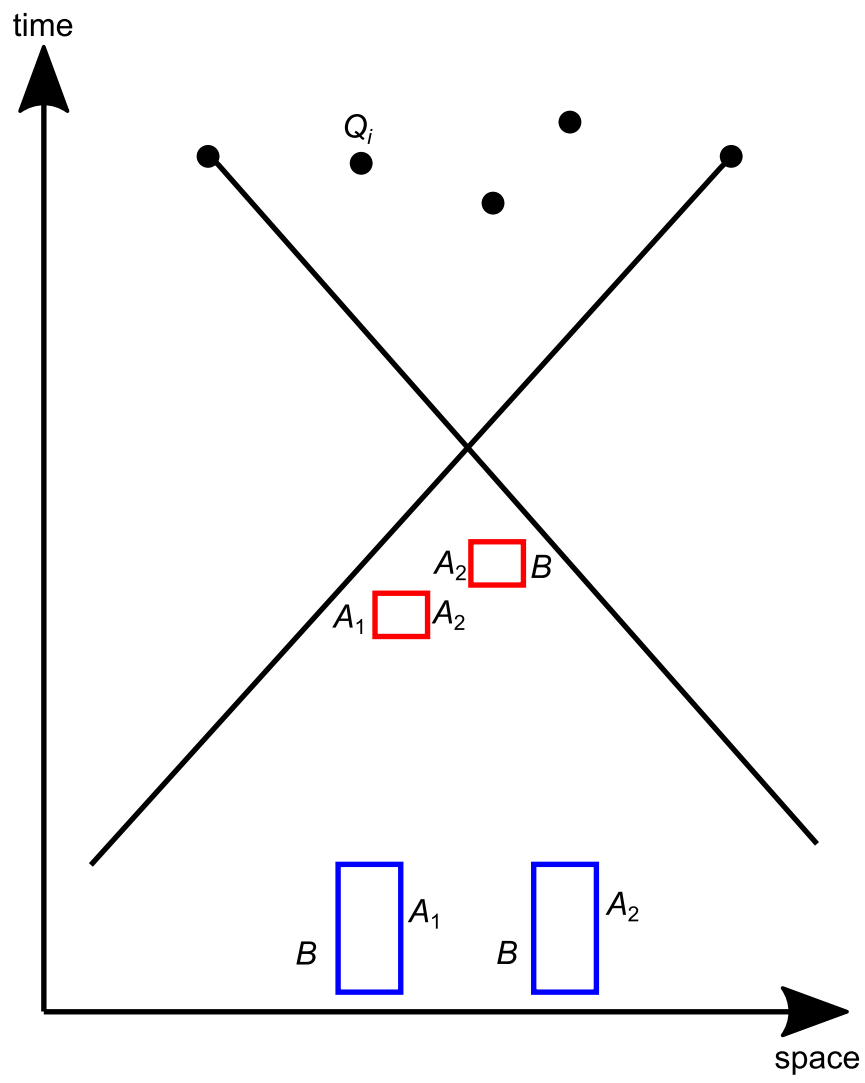

FIG. 2. Schematic illustration in 1+1-dimensional Minkowski spacetime of transfer of an S-money token in our flexible scheme. We use units in which the speed of light is unity. The black lines denote light rays. The bank sends quantum states to potential users $A_{1}, A_{2}, \ldots$ to initiate bit-string coordination protocols, which effectively commit these users to random classical bit strings $x_{1}, x_{2}, \ldots$ These protocols (represented by the large blue boxes) may be relatively lengthy but can be carried out well before tokens are generated or transferred. If $A_{1}$ initiates a token protocol (not illustrated), then she may later wish to transfer the token to another user $A_{2}$. This requires only classical communications (represented by the small red boxes) between local agents of $A_{1}$ and $A_{2}$ and between local agents of $A_{2}$ and $B$. Such communications can be completed relatively quickly, leaving $A_{2}$ in control of the token and free to make her own choice of a presentation point.

description of the subset $S_{2} \subset S_{1}$ committed using further data from their precommitted random string, and so on, with the valid presentation point finally decided and committed to at (or before) the final point $P_{D_{m}}$.

These commitments may be coded efficiently if the configuration of the decision points and the sizes and relations of the relevant subsets are suitable and known in advance. For example, if it is known in advance that a binary choice will be made at each successive decision point, selecting one of two equally sized known subsets, then the user may simply commit using successive bits of the string $x$ that would have been used to commit directly to $b$ in the unrefined protocol.

\section{A. Example}

In Fig. 3, we illustrate a simple example with eight presentation points $Q_{i}$, with $i \in S=\{0,1\}^{3}$, and two decision points, 


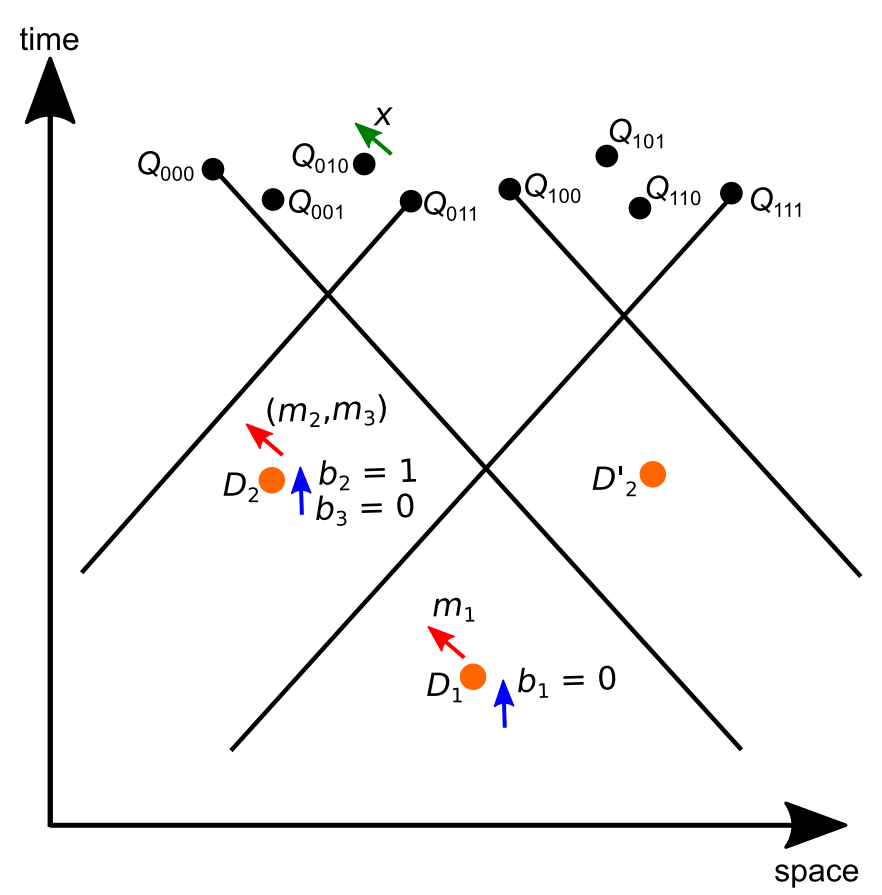

FIG. 3. The refinement allowing Alice to delay her chosen presentation point described in the main text is illustrated in $1+1$ dimensional Minkowski spacetime. We use units in which the speed of light is unity. In this example, there are eight presentation points (black dots). The black lines denote light rays. Alice has spacetime decision points $D_{1}$ and $D_{2}$ (orange dots). $D_{1}$ is in the causal past of all the presentation points. $D_{2}$ is in the causal past of $Q_{0 i_{2} i_{3}}$ for all $i_{2}, i_{3} \in\{0,1\}$. Alice's choices for the bit entries of the label $b=\left(b_{1}, b_{2}, b_{3}\right)=(0,1,0)$ of her chosen presentation point $Q_{b}$ are illustrated with the blue vertical arrows. The bit $m_{1}=x_{1} \oplus b_{1}$ and the two-bit string $\left(m_{2}, m_{3}\right)=\left(x_{2}, x_{3}\right) \oplus\left(b_{2}, b_{3}\right)$ that Alice sends Bob at $D_{1}$ and $D_{2}$, respectively, are indicated by the red diagonal arrows. Presentation of the token by Alice to Bob at $Q_{b}$ consists in Alice unveiling her three bit string $x$ to Bob at the presentation point $Q_{b}$, as illustrated with the green diagonal arrow. An alternative decision point, $D_{2}^{\prime}$, is also shown. Alice may use this if she chooses $b_{1}=1$ at $D_{1}$.

$N=2$. Let $x, b \in\{0,1\}^{3}$ and let $i_{k}$ be the $k$ th bit entry of $i$ for $k \in\{1,2,3\}$ and for $i \in\{x, b\} . D_{1} \equiv P_{D_{1}}$ is a spacetime point within the intersection of the causal pasts of all the presentation points, where Alice decides that her presentation point $Q_{b}$ will belong to the set $\left\{Q_{b_{1} 00}, Q_{b_{1} 01}, Q_{b_{1} 10}, Q_{b_{1} 11}\right\}$, i.e., with $S_{1}=\left\{\left(b_{1}, i_{2}, i_{3}\right): i_{2}, i_{3} \in\{0,1\}\right\}$. In this example, we suppose she chooses $b_{1}=0$. At $D_{1}$, Alice indicates to Bob that she has chosen her presentation point to have the bit $b_{1}$ fixed, without telling him the chosen value for $b_{1}$, and sends him the bit $m_{1}=x_{1} \oplus b_{1}$, where $x_{1}$ is the first bit of the previously committed string $x=\left(x_{1}, x_{2}, x_{3}\right)$. At the decision point $D_{2} \equiv P_{D_{2}}$, which is in the causal past of all $Q_{i}$ with $i \in S_{1}$, Alice chooses the bits $b_{2}$ and $b_{3}$ of her presentation point $Q_{b}$, with values $b_{2}=1$ and $b_{3}=0$ in this example, she indicates to Bob that she has chosen her presentation point and sends the two-bit string $\left(m_{2}, m_{3}\right)=\left(x_{2}, x_{3}\right) \oplus\left(b_{2}, b_{3}\right)$ to Bob. Alice presents her token at $Q_{b}$ by unveiling to Bob's agent at $Q_{b}$ her commitment to the string $x$. Bob's agent at $Q_{b}$ validates Alice's token at $Q_{b}$, with $b=\left(b_{1}, b_{2}, b_{3}\right)$, if he validates Alice's commitment to $x$ and if $m_{i} \oplus x_{i}=b_{i}$ for $i=1,2,3$.

\section{B. Comparison with previous S-money schemes}

Previous S-money schemes can also allow users to delay the choice of presentation point. For example, Ref. [7] considers a scheme in which the user may present the token at a presentation point $Q_{b}$ by giving an issuer's agent there her string $\mathbf{y}$ of measurement outcomes, or she may delay the presentation of her token by stating this to the issuer's agent at $Q_{b}$. In the latter case, the issuer's agent gives further quantum states to the user at $Q_{b}$, and the user measures the received quantum states at $Q_{b}$ in a basis labeling her chosen presentation point $Q_{b^{\prime}} \succ Q_{b}$, obtaining a string of measurement outcomes $\mathbf{y}^{\prime}$. At $Q_{b^{\prime}}$, the issuer may present the token by giving the issuer's agent there the string $\left(\mathbf{y}, \mathbf{y}^{\prime}\right)$, or she may choose to further delay the presentation point, and so on.

This method has the disadvantage that it requires various quantum communication exchanges between user's and issuer's agents at various spacetime points. This is technologically very demanding because the issuer and user's agents must have quantum devices to transfer and measure quantum states at all presentation points where the token presentation can be delayed. On the other hand, our scheme only requires the quantum communication between user and issuer to take place once, between a single pair of adjacent laboratories at a spacetime region $P$, so that delays of the presentation point are needed only to allow time for classical communications between user and issuer's agents at spacetime points in the causal future of $P$.

\section{DISCUSSION}

In this paper we have presented refinements of S-money schemes introduced in Refs. [6-9]. These refinements make the schemes considerably more flexible and practical. They allow the initial commitment phase, which involves quantum communications and measurements and potentially may be relatively lengthy, to take place long in advance. This allows the user to choose her presentation point at potentially any point in the intersection of the causal pasts of all the presentation points, limited only by the need to complete the commitment by relatively short classical communications. It also allows the user to make a series of decisions, which may be independent, that collectively determine the presentation point. This flexibility is valuable in applications where speed is critical. Further, it allows S-money tokens to be simply and efficiently transferred between users. The refinements retain the properties of unforgeability and future user privacy, both for the original user and for users to whom the token is transferred.

\section{ACKNOWLEDGMENTS}

The authors acknowledge financial support from the UK Quantum Communications Hub Grant No. EP/M013472/1. A.K. is partially supported by Perimeter Institute for Theoretical Physics. Research at Perimeter Institute is supported by the Government of Canada through Industry Canada and by the Province of Ontario through the Ministry of Research and Innovation. 
[1] S. Wiesner, ACM Sigact News 15, 78 (1983).

[2] D. Gavinsky, in Proceedings of the 2012 IEEE 27th Annual Conference on Computational Complexity (CCC'12) (IEEE, Los Alamitos, CA, 2012), pp. 42-52.

[3] F. Pastawski, N. Y. Yao, L. Jiang, M. D. Lukin, and J. I. Cirac, Proc. Natl. Acad. Sci. USA 109, 16079 (2012).

[4] M. Georgiou and I. Kerenidis, in Leibniz International Proceedings in Informatics, LIPIcs, Vol. 44 (Schloss Dagstuhl-LeibnizZentrum fuer Informatik, Dagstuhl, Germany, 2015).

[5] Depending on context, "S" may denote "summonable" or "super."

[6] A. Kent, Proc. R. Soc. A 475, 20190170 (2019).

[7] A. Kent, Quantum tokens, International Patent Application No. WO2017001850A1 (2017).

[8] A. Kent, Future position commitment, International Patent Application No. WO2017001850A1 (2018).

[9] A. Kent, Cryptographic method and system, UK Patent Application No. GB1719870.6 (2017).
[10] A. Kent, Phys. Rev. Lett. 109, 130501 (2012).

[11] S. Croke and A. Kent, Phys. Rev. A 86, 052309 (2012).

[12] J. Kaniewski, M. Tomamichel, E. Hänggi, and S. Wehner, IEEE Trans. Inf. Theory 59, 4687 (2013).

[13] T. Lunghi, J. Kaniewski, F. Bussières, R. Houlmann, M. Tomamichel, A. Kent, N. Gisin, S. Wehner, and H. Zbinden, Phys. Rev. Lett. 111, 180504 (2013).

[14] D. Pitalúa-García and I. Kerenidis, Phys. Rev. A 98, 032327 (2018).

[15] D. Pitalúa-García, Phys. Rev. A 100, 012302 (2019).

[16] A. Kent, D. Lowndes, D. Pitalúa-García, and J. Rarity, Practical quantum tokens without quantum memories: towards a proofof-principle experimental demonstration (unpublished).

[17] In practical implementations with current technology, the transmitted state will be a sequence of (approximate) qubits, and the measurement a sequence of individual measurements on these. We represent these as a single state and single collective measurement to simplify the notation. 